\title{
Korrespondenzen.
}

\section{Die Behandlung des Keuchhustens.}

\author{
Von Hofrat Dr. Stepp in Nürnberg.
}

In No. 41 dieser Wochenschrift hat Herr Prof. Dr. Feer einen bemerkenswerten Vortrag über Keuchhusten veröf fentlicht, welchem ich nach meinen vielfachen Erfahrungen nur beipflichten kann. Nur seine Ausführungen über die Anwendung des Bromoforms nötigen mich, um meine Priorität zu wahren, zu einigen Bemerkungen.

Herr Prof. Dr. Feer schreibt: „Die Dosierung richte ich nach den Angaben von Fiertz: etc." Nun habe ich als Erster das Bromoform in die Praxis eingeführt und ich habe im Jahre 1889 die erste Mitteilung in dieser Wochenschrift, No. 31, über die Anwendung des Bromoforms bei Keuchhusten und die Dosierung desselben gegeben. ${ }^{1}$ ) Herr Fiertz hat im Jahre 1894 in einer Inauguraldissertation meine Angaben mit geringer Modifikation reproduziert.

Dann habe ich im Jahre 1895 in No. 36 der Münchner medizinischen Wochenschrift über die Wirkung des Bromoforms bei Affektionen der Atmungsorgane im allgemeinen und insbesondere bei Keuchhusten Mitteilungen gegeben. Bei dieser Gelegenheit möchte ich besonders auf die überaus günstige Wirkung des Bromoforms bei dem Katarrh der Emphysematiker und das begleitende Asthma (viermal täglich 0,5 in Kapseln) nochmals dringlich hingewiesen haben.

Endlich weise ich noch auf einen weiteren Artikel von mir in der Prager medizinischen Wochenschrift 1903, No. 11-14 hin, in welchem ich mich gegen die narkotische Wirkung des Bromoforms, wie sie Herr Prof. Dr. Feer annimmt, ausgesprochen habe. Dort heißt es: Ich habe wiederholt die Beobachtung gemacht, daß bei Vergiftungsfällen, die glücklich abliefen, der Keuchhusten von Stunde an geheilt war. Diese plötzliche Heilung nach so enormen Dosen bei Vergiftungsfällen würde unverständlich sein, wenn nicht eine spezifische Wirkung auf den Keuchhustenerreger stattfände, denn das Bromoform wird durch die Lungen ausgeschieden.

Nun muß ich auf Grund meiner Erfahrungen feststellen, daß es, allerdings sehr vereinzelt, kindliche Naturen gibt, welche eine Idiosynkrasie gegen Bromoform haben und bei welchen auch geringe Gaben narkotisch wirken. Bei solchen Kindern muß man von Bromoform absehen! Denn es wird bei weiterer Anwendung nichts erreicht, und nur die narkotischen Erscheinungen - ohne Verminderung der Anfälle! - treten in den Vordergrund. Aber bei der überwiegend großen Anzahl der Kinder ist das nicht der Fall, das Mittel wird vortrefflich vertragen und kommt zur vollen Geltung, die Kinder sind munter, haben besseren Appetit wie früher, die Ernährung nimmt zu, und die kleinen Patienten sehen nach der Krankheit besser aus, als dies vorher der Fall war.

Seit 19 Jahren hat sich das von mir in die Praxis eingeführte Bromoform in der von mir festgestellten Anwendung bewährt, und ich freue mich, in Herrn Prof. Dr. Feer einen so gewichtigen Beurteiler gefunden zu haben. 